\title{
Adherence in severe asthma: time to get it right
}

\author{
Vanessa M. McDonald ${ }^{1,2}$ and Janelle Yorke 3,4
}

Affiliations: ${ }^{1}$ Priority Research Centre for Healthy Lungs and Centre of Excellence in Severe Asthma, Faculty of Health and Medicine, The University of Newcastle, Newcastle, Australia. ${ }^{2}$ Dept of Respiratory and Sleep Medicine, John Hunter Hospital, Newcastle, Australia. ${ }^{3}$ School of Health Sciences, Faculty of Biology, Medicine and Health, University of Manchester, Manchester, UK. ${ }^{4}$ Manchester Academic Health Sciences Centre, University of Manchester, Manchester, UK.

Correspondence: Vanessa M. McDonald, Centre of Excellence in Severe Asthma and Priority Research Centre for Healthy Lungs, The University of Newcastle, 1 Kookaburra Circuit, New Lambton Heights, 2305, NSW, Australia. E-mail: Vanessa.McDonaldanewcastle.edu.au

@ERSpublications

Adherence in severe asthma is suboptimal as are the clinic measures to assess adherence http://ow.ly/CSZH30gCsBQ

Cite this article as: McDonald VM, Yorke J. Adherence in severe asthma: time to get it right. Eur Respir J 2017; 50: 1702191 [https://doi.org/10.1183/13993003.02191-2017].

How many times have you sat in an asthma consultation and asked a patient "how many doses of your inhaler have you forgotten to take in that last week?" There are times when you may understandably question the person's response, and other times when you will be convinced that they "take their puffers without fail" only to subsequently discover that you have been mistaken. Whilst clinicians want to understand the individual's level of treatment adherence, this approach is clearly flawed and we need to do better.

Suboptimal treatment adherence is common in severe asthma, in both children and in adults, with reports of suboptimal adherence in $>50 \%$ of individuals with asthma [1]. There are multiple reasons that people with severe asthma either choose not to take treatment as prescribed or are unintentionally nonadherent [2]. Whether treatment nonadherence stems from a conscious decision not to take the treatment, an erroneous understanding or use of the treatment, or simple forgetfulness, the goals remain the same. Suboptimal treatment adherence in asthma leads not only to poor control, increased risk of asthma attacks and subsequently, increased healthcare utilisation [3,4], but it frequently results in unnecessary escalation of treatments, which can be both toxic and expensive. Adherence to asthma treatments by children is further complicated by their dependence on parents (or carers) for the provision and often administration of asthma therapy.

Thus, there is a number of things that we need to get right: we need to be able to identify suboptimal adherence in individuals with severe asthma, understand the reasons for their behaviour, and, importantly, design, test and implement treatment approaches aimed at improving and maintaining adherence to asthma medication. The importance of this has recently been highlighted in the Lancet Commission "After Asthma: Redefining Airways Disease" [5], in which treatment adherence and management of are reinforced in the key recommendation "Make the most of new treatment opportunities in severe disease" [5].

Another challenge in severe asthma is determining where individuals fit in the classification schema of difficult-to-treat asthma and severe treatment-refractory asthma $[1,6]$. The underlying challenges of

Received: Oct 252017 | Accepted after revision: Oct 262017

Conflict of interest: Disclosures can be found alongside this article at erj.ersjournals.com

Copyright OERS 2017 
difficult-to-treat asthma usually relate to suboptimal adherence and self-management skills, and unaddressed comorbidities, triggers and aggravating factors [7]. Each of these asthma subtypes requires a different approach to management. Difficult-to-treat asthma may respond to a multidisciplinary, multidimensional assessment to correct these traits [8], where as individuals with severe treatment refractory asthma may benefit from further phenotyping and endotyping $[7,9,10]$ and additional targeted therapies. Identifying reliable ways to distinguish these patient groups is of utmost importance in order to personalise treatment approaches and ensure that each individual is prescribed the right treatment at the right time [11]. Furthermore, strategies that can be readily used in the clinic to assess and quantify suboptimal adherence, and to improve behaviour are also needed in severe asthma, to ensure not only that the right individual is prescribed the right treatment at the right time, but that they indeed also receive it!

As reported in this issue of the European Respiratory Journal, Jochmann et al. [12] sought to determine whether electronic monitoring in children with persistent asthma could differentiate individuals with severe treatment-refractory asthma from those with difficult-to-control asthma, and to assess whether electronic monitoring of asthma could improve treatment adherence. They report a prospective observational study involving 93 children with asthma, aged 5-17 years. The children were assessed at baseline and at follow up between 8 and 16 weeks. The follow up times varied as visits were embedded into clinical assessments.

After optimisation of inhaler technique, the children underwent a period of adherence monitoring using electronic monitoring devices that recorded the time and date of inhaler doses activated. Children also completed an array of assessments at baseline and follow-up to measure asthma control, quality of life, lung function, exhaled nitric oxide fraction $(\mathrm{FeNO})$ and adherence questionnaires.

A priori, the investigators defined "good" adherence as the use of $>80 \%$ of the prescribed doses, between $60 \%$ and $79 \%$ was "moderate", and $<60 \%$ as "poor". The study confirms suboptimal adherence in children with asthma, as good adherence was only evident in $42 \%$ of the population and suboptimal in the remainder. Moreover, there were no baseline clinical parameters that could distinguish those that were adherent from those that were not, reinforcing the difficulty of establishing these behaviours in the clinical setting.

Electronic monitoring could distinguish those with likely severe treatment-refractory asthma from those with difficult asthma related to suboptimal adherence. Given the challenges of doing this in the clinic, this is a clinically important observation.

Whilst the use of objective measures to assess adherence had increased in research settings, use and access to suitable methods in clinical practice remains limited. Self-report is frequently used in this setting, but this approach will under estimate adherence. As in this study, where all families indicated that they used their prescribed treatments most of the time and as instructed, but the use of objective monitoring proved otherwise in $58 \%$ of cases.

In some countries, clinicians can access prescription records and this approach is frequently used; however, these data from Jochmann et al. [12] demonstrate the limitations of this approach. There was no correlation $(\mathrm{r}=0.286, \mathrm{p}=0.051)$ between electronic monitoring and records for uptake of prescription. Furthermore, adherence defined by electronic monitoring was as low as $27 \%$ in individuals that had $100 \%$ prescription uptake.

Electronic monitoring, as the authors have shown, can identify individuals with suboptimal adherence and can help distinguish those with likely severe treatment-refractory asthma from difficult-to-treat asthma related to suboptimal adherence. However, again, these devices are not without limitation: cost can be an issue, they do not assess nonadherence due to poor technique, and even when patients are aware that they are being monitored, suboptimal adherence remains.

JochmanN et al. [12] acknowledge this and highlight another important need, which is to determine interventions that lead to behaviour change. The observational design of this study did not allow this hypothesis to be tested but it did show that those with good adherence and good asthma control were the group that achieved improvements in a range of measures at follow-up, including forced expiratory ratio in $1 \mathrm{~s}, \mathrm{FeNO}$, quality of life, exacerbations and percentage of bronchodilator reversibility. In order to progress further, interventions designed to not only promote adherence but also ensure adherence is maintained are needed. To design interventions such as this, a better understanding of the experiences and beliefs of children, families and adults with severe asthma is needed. These should not be extrapolated from studies of individuals with mild-to-moderate asthma, as we acknowledge that severe asthma is a different disease, not only from a medical pathophysiological perspective $[1,13]$ but also from a patients' perspective [2]. This is due to the symptom and exacerbation burden, additional treatment requirements and the impact on work, school and family life. A better understanding of the experience of individuals could help inform the design of such interventions. 
There have been advances been made in severe asthma management with the introduction of new biological agents, these agents lead to reduced exacerbations when given to the right patient [14, 15]. There are patients with severe asthma who, despite good adherence, will remain refractory to standard treatment and will likely benefit for these targeted therapies [15], but before escalating treatments, we must surely try to get the basics right.

Adherence in asthma is challenging and frustrating, but achieving better adherence is a necessary goal. We must accept that suboptimal adherence is the norm, and become more objective in our assessment of adherence and seek ways to get it right.

\section{References}

1 Chung KF, Wenzel SE, Brozek JL, et al. International ERS/ATS guidelines on definition, evaluation and treatment of severe asthma. Eur Respir J 2014; 43: 343-373.

2 Foster JM, McDonald VM, Guo M, et al. "I have lost in every facet of my life": the hidden burden of severe asthma. Eur Respir J 2017; 50: 1700765.

3 Pike KC, Levy ML, Moreiras J, et al. Managing problematic severe asthma: beyond the guidelines. Arch Dis Child 2017 in press [https://doi.org/10.1136/archdischild-2016-311368].

4 McGrady ME, Hommel KA. Medication adherence and health care utilization in pediatric chronic illness: a systematic review. Pediatrics 2013; 132: 730-740.

5 Pavord ID, Beasley R, Agusti A, et al. After asthma: redefining airways diseases. Lancet 2017 in press [https://doi. org/10.1016/S0140-6736(17)30879-6].

6 Bush A, Saglani S, Fleming L. Severe asthma: looking beyond the amount of medication. Lancet Respir Med 2017; 5: $844-846$.

7 McDonald VM, Maltby S, Reddel HK, et al. Severe asthma: current management, targeted therapies and future directions - a roundtable report. Respirology 2017; 22: 53-60.

8 Clark VL, Gibson PG, Genn G, et al. Multidimensional assessment of severe asthma: a systematic review and meta-analysis. Respirology 2017; 22: 1262-1275.

9 Agusti A, Bel E, Thomas M, et al. Treatable traits: toward precision medicine of chronic airway diseases. Eur Respir J 2016; 47: 410-419.

10 Agusti A, Bafadhel M, Beasley R, et al. Precision medicine in airway diseases: moving to clinical practice. Eur Respir J 2017; 50: 1701655.

11 Gibson PG, Marks G, Sly P, et al. Case for Action - Proposal to NHMRC: Targeted Therapy for Asthma. Canberra, NHMRC, 2015.

12 Jochmann A, Artusio L, Jamalzadeh A, et al. Electronic monitoring of adherence to inhaled corticosteroids: an essential tool in identifying severe asthma in children. Eur Respir J 2017; 50: 1700910.

13 Wenzel SE, Brillhart S, Nowack K. An invisible disease: severe asthma is more than just "bad asthma". Eur Respir J 2017; 50: 1701109.

14 Yancey SW, Ortega HG, Keene ON, et al. Meta-analysis of asthma-related hospitalization in mepolizumab studies of severe eosinophilic asthma. J Allergy Clin Immunol 2017; 139: 1167-1175.

15 Grainge CL, Maltby S, Gibson PG, et al. Targeted therapeutics for severe refractory asthma: monoclonal antibodies. Expert Rev Clin Pharmacol 2016; 9: 927-941. 